\title{
Análisis de la integración de la bioética en la investigación de enfermería ${ }^{1}$
}

Institución: Universidad de Costa Rica

Viriam Leiva Díaz ${ }^{2}$ Beatriz Villalobos Núñez ${ }^{3}$

\section{COMO CITAR}

Leiva, V. y Villalobos, B. (2015). Análisis de la integración de la bioética en la investigación de enfermería. Rev. Enfermería Actual en Costa Rica, 28, 1-19. DOI: http://dx.doi.org/10.15517/revenf.v0i28.17061http://dx.doi.org/10.15517/revenf.v0i28.17061

\section{RESUMEN}

Introducción. El objetivo de este artículo es presentar los resultados del análisis de los Trabajos Finales de Graduación (TFG), desarrollados por el estudiantado como requisito para obtener el título de Licenciatura en Enfermería, los cuales son parte del plan de estudios del Programa de Licenciatura en Enfermería de la Universidad de Costa Rica.

Método. La indagatoria se ejecutó por medio de una investigación bibliográfica, documental, archivista, de la que se analizó 158 Trabajos Finales de Graduación producidos entre el 2005 y el año 2012. De las memorias e informes finales de investigación se extrajo aspectos que reflejaran implícita o explícitamente los principios de la bioética por medio de una matriz elaborada para ese fin.

Resultados. A pesar de que la población objeto de estudio o intervención estuvo representada por grupos vulnerables, en un número importante de los TFG, no se documentó adecuadamente el proceso de consentimiento informado, además de que en el apartado de metodología no se evidencia con claridad la forma en que se aseguró los principios bioéticos.

Conclusión. Se debe trabajar con mayor intensidad y claridad en el proceso de aprendizaje del estudiantado acerca de cuán importante son los principios bioéticos, al punto de que debe explicitarse el cómo se respetaron.

Palabras clave: Bioética, enfermería, investigación.

\footnotetext{
${ }^{1}$ Fecha de recepción: 27 de marzo, 2014

Fecha de aceptación: 29 de abril, 2014

${ }^{2}$ Universidad de Costa Rica. Escuela de Enfermería. Costa Rica. Correo electrónico: viriaml@gmail.com

${ }^{3}$ Universidad de Costa Rica. Escuela de Enfermería. Costa Rica. Correo electrónico: bvillaenf@yahoo.es
} 


\title{
Analysis of the integration of bioethics in nursing research ${ }^{1}$
}

\author{
Viriam Leiva Díaz ${ }^{2}$ \\ Beatriz Villalobos Núñez ${ }^{3}$
}

Institution: University of Costa Rica

\section{CITED:}

Leiva, V. y Villalobos, B. (2015). Analysis of the integration of bioethics in nursing research. Rev. Enfermeria Actual en Costa Rica, 28, 1-19. DOI: http://dx.doi.org/10.15517/revenf.v0i28.17061

\begin{abstract}
Introduction. This article claims to present the results of an analysis regarding the final graduation projects made by students as a requisite for the Licentiate Degree in Nursing, as a part of the Degree Program in Nursing in the University of Costa Rica.

Method. The study was carried out by means of a bibliographical and documental investigation of 158 final graduation projects, produced between the years 2005 and 2012. Characteristics which reflected implicitly or explicitly the principles of bioethics were extracted from the final reports, by means of a base by date created for this purpose.

Results. It is noted that although in an important number of the final graduation projects the studied populations were vulnerable groups, the informed consent process is not adequately documented. Similarly, it is not clear the way in which the bioethics principles were ensured.

Conclusion. It is concluded that there must be more intense work and clarity throughout the student's learning process, regarding the importance of respecting the principles of bioethics, showing explicitly the way in which they were respected.
\end{abstract}

Key words: Bioethics, nursing, research.

\footnotetext{
${ }^{1}$ Date of receipt: March 27, 2014

Date of acceptance: April 29, 2014

${ }^{2}$ University of Costa Rica. School of Nursing. Costa Rica. E-mail: viriaml@gmail.com

${ }^{3}$ University of Costa Rica. School of Nursing. Costa Rica. E-mail: bvillaenf@yahoo.es
} 


\section{INTRODUCCIÓN}

El interés de este artículo no es iniciar una disertación acerca de la historia y conceptualización de la bioética, tema ya bastante discutido (Gracia, 1989; Garrafa, 1999, Garrafa, Do Prado, 2001; Hottois, 2007; Vidal, 2010). En este caso, tan solo se menciona el origen de la palabra bioética, cuyas raíces griegas "bios" (vida) y "ethos" (comportamiento o conducta), en conjunto, se refieren al análisis de los problemas éticos que se gestan en la relación del ser humano con las ciencias y la tecnología, los cuales pueden influir o modificar la vida de la persona.

Considerando lo anterior, la bioética debe ser comprendida como una disciplina del saber que se reduce únicamente a la reflexión ética de principios que guían un saber específico o la reflexión ética clínica de un saber en un entorno específico hospitalario o no; debe trascender al entendimiento hasta llegar a la reflexión del impacto que produce la ciencia junto con el avance tecnológico que, en muchas ocasiones, priva a las personas de la relación empática, cálida, es decir, efectiva y afectiva, que despoja al individuo de ser partícipe en la toma de decisiones sobre su derecho a la vida, a la salud y a la muerte.

Visto desde esa perspectiva, la bioética tiene algo que decir en la enfermería como ciencia de la salud, al tener esta precisamente como objeto de estudio el cuidado en el que se incluye, tal como lo mencionan Daza y Medina (2006), cinco líneas de significado básico, denominadas así por las autoras; dichas líneas son el "cuidado como rasgo humano, Cuidado como imperativo moral, Cuidado como interacción personal, Cuidado como afecto, Cuidado como intervención terapéutica"(p. 56). Estos significados básicos reflejan no solo el cuidado como una función inherente a la persona, sino como un imperativo moral, ético, en una relación humanizada y holística, y finalmente, como fin último del accionar del profesional de enfermería, quien debe aprender estos componentes durante su formación profesional, que no tiene que ver únicamente con la realización de acciones o técnicas de soporte del cuidado terapéutico, sino con la dimensión científica.

Esta dimensión está inscrita en uno de los cuatro patrones o formas de conocer de la enfermería propuestos por Carper (1978), como el patrón empírico que, según Durán (2002) es "la teoría científica, los modelos para la práctica, la explicación y predicción de hechos" ( p.11) y expresa además que "la investigación, el desarrollo teórico y la práctica basada en el conocimiento, obtenido a través de los dos primeros, son los ejes alrededor de los cuales gira la epistemología de la enfermería, y por ese motivo debemos poner énfasis en este tipo de desarrollo teórico" (p.13).

Partiendo de lo anterior, se afirma que la investigación no es un anexo en la enfermería, un apéndice que puede o no estar presente, sino que es el sustento de la reflexión del ser, saber y hacer enfermero, lo cual se explicita en la siguiente cita: 


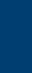

(

\begin{abstract}
¿Por qué cuando se habla de la epistemología de la enfermería se debe, forzosamente, mirar la tríada teoría-práctica-investigación? La respuesta es simple [...] la razón de ser de la enfermería es la práctica profesional e, idealmente, todo conocimiento de enfermería debe tender a solucionar los problemas de la práctica. Por esta razón, la misión de la investigación que genera, redefine o produce nuevo conocimiento es desarrollar teorías que describan, expliquen, predigan y prescriban el comportamiento de los fenómenos de la enfermería (Durán, 2002, p.13).
\end{abstract}

Entonces, en el proceso de mediación educativa es fundamental que no solo se enseñe las técnicas prácticas del hacer de enfermería, sino que se le brinde al estudiantado los elementos necesarios para el desarrollo de la investigación como elemento sustantivo de la enfermería (Sanhueza, 2009), a lo que hay que agregar la enseñanza de los principios éticos otro de los patrones del conocimiento mencionado por Carper (1978), el cual se refiere al conocimiento moral enfermero, no solo en lo que respecta al cuidado o la relación con la persona usuaria del cuidado terapéutico, sino de la persona que participa en un proceso investigativo en enfermería, aspectos que se reflejan en la rigurosidad investigativa, así como en la práctica de los principios éticos, códigos, normativas y estándares dentro de la investigación.

Los resultados que se presentan en este proyecto de investigación, \#421-B1-331, aprobado por la Vicerrectoría de la Universidad de Costa Rica, se relaciona con lo anterior y su objetivo fue analizar la implementación de la bioética en la investigación en enfermería, específicamente en los Trabajos Finales de Graduación, en los que se supone que es en el que se plasma el proceso formativo de los estudiantes de la Licenciatura en Enfermería de la Universidad de Costa Rica.

\section{MATERIALES Y MÉTODOS}

Para el proceso del estudio se planteó la investigación bibliográfica, documental, archivista, caracterizada por la revisión de documentos que se encuentran en archivos (Torre y Navarro, 1982); en cuanto a los instrumentos bibliográficos, se usó las memorias o informes finales de los Trabajos Finales de Graduación (TFG) elaborados por los estudiantes de la carrera de Licenciatura en Enfermería, trabajos que fueron defendidos y publicados en el periodo 2005 -2012. Este tipo de investigación consta de pasos o fases claramente establecidos que se describen a continuación.

Para la primera fase, localizar información, se efectuó búsquedas en el SIBDI (Sistema de Bibliotecas, documentación e información), así como en el CESISA (Centro de Simulación en Salud) para detectar los trabajos escritos de las diferentes modalidades de graduación, de modo que se accediera a ellos de forma física o digital. En total, se obtuvo $154 \mathrm{TFG}$, la distribución por modalidad de graduación se presenta en el apartado de resultados.

Seguidamente, la consolidación de la información obtenida, se realizó mediante el vaciamiento en una base de datos, elaborada para tal efecto. 
Para el análisis de los documentos, fuentes o datos, se elaboró las fichas de contenido textual, lo cual ha ayudado contribuido con el manejo de los datos de los cursos y con la elaboración del primer borrador de la base de datos, para lo que se respetó los siguientes criterios establecidos para su revisión:

Información general: nombre del proyecto, año de ejecución, número de estudiantes que participaron, modalidad, lugar de desarrollo del TFG y población meta.

Información acerca de la integración de aspectos bioéticos: presencia de descripción de los aspectos bioéticos en la metodología; aspectos bioéticos tomados en cuenta en el desarrollo del trabajo; mención de aspectos bioéticos como confidencialidad de la información y aplicación del principio de autonomía; mención de limitaciones o regulaciones para salvaguardar la bioética en el desarrollo del trabajo.

Información acerca de la integración del Consentimiento Informado: presencia de Consentimiento Informado (CI) en anexos, uso de CI de la Vicerrectoría de Investigación o mención de la fuente; presencia del CI adaptado para niños y niñas o grupos vulnerables.

Dichos criterios cumplen con el fin de analizar la presencia implícita o explícita de los aspectos mencionados en el epígrafe previo en los diferentes apartados del informe final de graduación; de igual forma estos sirvieron como hilos conductores que permitieron localizar y seleccionar la información de una manera fácil y precisa.

La organización de estos contenidos, así como la revisión esquemática permitió verificar si el proceso de investigación es acertado y correcto, así como valorar el material recopilado, la localización de posibles lagunas, detección de excesos en las ideas transcritas, con el objetivo de organizar y brindar mayor uniformidad a la investigación y para determinar si faltaban datos esenciales.

Al finalizar el proceso anterior, fue posible clasificar el material recopilado y depurar aquellos datos con mayor valor para responder al objetivo de estudio, de lo que se obtuvo un esquema más perfeccionado que el anterior y que se presenta en el apartado de resultados del presente artículo.

\section{Consideraciones bioéticas}

Para el desarrollo de la investigación cuyos resultados se ofrecen en forma sucinta, para lo interno, en el presente artículo se tomó en cuenta una estructura ética que permitiera ofrecer con claridad y veracidad la información, siempre apegada al método de investigación seleccionado. De igual forma, se respetó los derechos de autor, entendiéndose como autoría la Escuela de Enfermería de la Universidad de Costa Rica y las personas que crearon los TFG, razón por la que se brinda las correspondientes citas bibliográficas en los casos que lo requieran. Como parte de dichas consideraciones, se omite el nombre de las personas que desarrollaron cada uno de los TFG. 


\section{RESULTADOS}

Tal como se mencionó, fue necesario establecer una serie de criterios para identificar y clasificar los resultados del estudio, los cuales sirvieron como guía para presentar la información.

En la tabla resumen 1, se brinda los datos generales de los 158 informes finales o memorias de graduación realizados entre los años 2005 y el 2012, los cuales se relacionan con la introducción

Tabla 1

Modalidad de trabajos finales de graduación según año y modalidad, 2005-2012

\begin{tabular}{cccccc} 
Año & Prácticas dirigidas & Tesis & Seminarios & Proyectos & Total \\
2005 & 6 & 4 & 5 & 2 & 17 \\
2006 & 10 & 3 & 2 & 1 & 16 \\
2007 & 12 & 3 & 3 & 1 & 19 \\
2008 & 12 & 2 & 2 & 0 & 16 \\
2009 & 10 & 3 & 1 & 0 & 14 \\
2010 & 22 & 5 & 2 & 4 & 33 \\
2011 & 21 & 5 & 2 & 5 & 33 \\
2012 & 0 & 3 & 6 & 1 & 10 \\
Total & 93 & 28 & 23 & 14 & 158 \\
Porcentaje $\left(h_{i}\right)$ & $59 \%$ & $18 \%$ & $14 \%$ & $9 \%$ & $100 \%$ \\
\hline
\end{tabular}

Fuente: elaboración propia.

Como se observa en el cuadro anterior, en el año 2010 y 2011 se desarrolló la mayor cantidad de trabajos finales de graduación, mientras que en el año 2012 se produjo la menor cantidad, lo cual se explica porque es a principios de cada año que el estudiantado puede defender públicamente su trabajo de graduación y el cohorte de la recolección de los datos fue en el primer semestre de ese año.

En cuanto a la modalidad, la de mayor preferencia es la práctica dirigida que, durante esos seis años, representó más de la mitad $\left(h_{i=5} 59 \%\right)$ de los trabajos finales de graduación realizados, seguido por la modalidad de tesis $\left(h_{i}=28 \%\right)$ y de seminario $\left(h_{i}=23 \%\right)$ con similar cantidad.

En lo referente al estudio, se ofrece información general necesaria para la comprensión e implicaciones en bioética y derechos humanos, la cual se presentará según la modalidad de Trabajo Final seleccionado por los estudiantes, la cual se brinda según mayor frecuencia y predilección en el alumnado.

Los lugares en que se desarrollaron las prácticas dirigidas son muy diversos, sin embargo se puede agrupar según el tipo de organización o institución, tal como se observa en la tabla 2. 


\section{Tabla 2}

\section{Lugares donde se llevaron a cabo las prácticas dirigidas, 2005- 2012}

\begin{tabular}{lc}
\hline \multicolumn{1}{c}{ Lugar } & Frecuencia $\left(\boldsymbol{f}_{\boldsymbol{i}}\right)$ \\
Hospitales C.C.S.S. & 18 \\
Centros o programas de I y II niveles de atención & 6 \\
Centros o programas para la atención de PAM ${ }^{2}$ & 14 \\
Organizaciones No gubernamentales & 20 \\
Instituciones educativas & 15 \\
Otras instituciones & 13 \\
Empresas privadas & 4 \\
Comunidades & 3 \\
Total $\left(\mathbf{F}_{\mathbf{i}}\right)$ & $\mathbf{9 3}$ \\
\hline
\end{tabular}

Fuente: elaboración propia.

Como se observa, en primer lugar se encuentran las organizaciones no gubernamentales $\left(f_{i}=20\right)$ como el lugar predilecto por los estudiantes para realizar las Prácticas Dirigidas ,seguidas por los hospitales y los centros de salud del I y II (24), las instituciones educativas tanto de primaria, secundaria como universitaria $\left(f_{i}=15\right)$. Finalmente, en otras instituciones se ubica el mayor número en importancia de TFG, compuesta por el Patronato Nacional de la Infancia (PANI), Cruz Roja Costarricense, diversas municipalidades, ministerios, centros de adaptación social (cárceles) y cooperativas.

En cuanto a la población meta ${ }^{3}$, con quienes se desarrolló más actividades fue con las cuidadoras (es) de personas que padecen alguna enfermedad $\left(f_{i}=12\right.$ trabajos $)$, seguida de las personas adolescentes $\left(f_{i}=11\right)$, las personas adultas mayores $\left(f_{i}=10\right)$, y $f_{i}=10$ con trabajadores (de distintas instituciones). En nueve trabajos, fue población meta, tanto el personal de enfermería (en diferentes instituciones y hospitales) como mujeres adultas; en $f_{i}=8$ estudios participaron personas adultas -como estudiantes- con algún padecimiento (desde preescolar hasta universidad); en siete casos, participaron los familiares de personas con algún padecimiento; en cinco; hombres adultos; en cuatro; $f_{i}=1$ infantes y personas con trastornos mentales; en tres, personas recluidas en centros penitenciarios; en dos casos, familias, y finalmente se tuvo una práctica en que se trabajó con mujeres trabajadoras sexuales, una con personas transgénero, y otra con docentes.

Toda esta amplia gama de poblaciones con las que se llevaron a cabo las 93 prácticas evidencian los focos que llaman más la atención de los estudiantes del grado de Licenciatura, sensibilizados de las necesidades de las poblaciones que eligen a partir de sus propias experiencias clínicas. Sin embargo, esta sensibilización debe incluir

\footnotetext{
${ }^{1}$ C.C.S.S.: Caja Costarricense del Seguro Social.

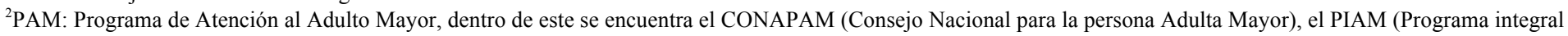
del Adulto Mayor), AGECO (Asociación Gerontológica Costarricense) entre los más importantes.

${ }^{3}$ Las prácticas dirigidas incluyen variedad de población para un mismo estudio, por ejemplo: usuarios de los servicios de salud y sus cuidadores (as).
} 
la interiorización de los aspectos bioéticos, de modo que se asegure la práctica de la bioética en cada una de las situaciones de enfermería, aspecto que se presenta a continuación.

Del total de prácticas dirigidas $\left(\mathrm{F}_{i}=93\right), f_{i}=34$ de ellos no incluyeron un apartado sobre "aspectos bioéticos" en la metodología, sin embargo, se mencionó algunos de estos aspectos en otras partes del trabajo.

Los aspectos bioéticos tomados en cuenta en el total de las prácticas dirigidas fueron principalmente el aseguramiento de la confidencialidad que fue tomada en cuenta en más de la mitad $\left(f_{i}=61\right)$, además se tramitó la autorización por parte de las instituciones donde se realizaron los estudios en un poco menos de la mitad $\left(f_{i}=38\right)$, el respeto a la autonomía de la persona se identificó en $f_{i}=13$ de los trabajos, y únicamente en $f_{i}=15$ casos del total se menciona la aplicación de los principios de beneficencia, no maleficencia y justicia.

Algunos trabajos aislados $\left(f_{i}=2\right)$ incluyen el compromiso de devolver los datos, así como que se tomó en cuenta la voluntariedad de participación en el estudio.

En cuanto al Consentimiento Informado (CI) estuvo, sí aparece en la memoria de la Práctica Dirigida en más de las tres terceras partes $\left(f_{i}=64\right)$ de los trabajos, y en $f_{i}=4$ de ellos, en la metodología, se indica que lo solicitaron de manera únicamente verbal. De los trabajos con CI escrito, menos de la mitad $\left(f_{i}=25\right)$ formuló un consentimiento ya estructurado, sea de la Vicerrectoría de Investigación de la Universidad de Costa Rica o de otra institución.

Cabe resaltar que en la mayoría de los trabajos este-CI-se dio como un encabezado en un cuestionario, en $f_{i}=31$ de ellos no se anexaron al trabajo aunque en algunos casos ( 1 de ellos) se mencionó en el texto su existencia escrita. Sólo en 9 del total de Prácticas Dirigidas se realizaron modificaciones al CI adaptándolo para niños (as), adolescentes (por ejemplo solicitando también autorización a los padres para que participen en el estudio), o grupos vulnerables como el de adultos mayores, personas con trastornos mentales, o con discapacidad visual, a pesar de que $f_{i}=37$ de los trabajos lo ameritaban, puesto que se llevaron a cabo con alguna de dichas poblaciones. En los casos en los que se modificó el $\mathrm{CI}$, en $f_{i}=2$ casos fueron eliminadas secciones del CI original que debían estar presentes.

En segundo lugar de elección de modalidad de graduación por parte del estudiantado, se encuentra la tesis, respecto de la cual se presenta los lugares en los que se realizó la investigación en la tabla 3. 


\section{Tabla 3}

\section{Lugares donde se llevó a cabo las tesis 2005- 2012}

\begin{tabular}{lc}
\hline \multicolumn{1}{c}{ Lugar } & Frecuencia $\left(f_{i}\right)$ \\
Hospitales C.C.S.S. & 10 \\
Centros o programas de I y II niveles de atención & 4 \\
Centros o programas para la atención de PAM & 1 \\
Familias en su domicilio & 3 \\
Instituciones educativas & 5 \\
Centros de reclusión & 2 \\
Centros de rehabilitación de adicciones & 1 \\
Internacionales (Suecia) & 1 \\
Hoteles & 1 \\
TOTAL $\left(\mathbf{F}_{\mathbf{i}}\right)$ & $\mathbf{2 8}$ \\
\hline
\end{tabular}

Fuente: elaboración propia.

La mitad de las investigaciones se desarrollaron en los hospitales y los centros de I y II nivel de atención en salud $\left(f_{i}=14\right)$, seguidos por las instituciones educativas $\left(f_{i}=5\right)$, mientras que el resto de trabajos investigativos fueron con familias en su domicilio, en centros de reclusión (cárceles).

En cuanto a la población meta elegida para las tesis, siete incluyeron a profesionales de enfermería; cinco, a la persona adolescente; cuatro, a niños o niñas, y en dos trabajos se estudió personal de enfermería en general, personas privadas de libertad, familias y personas adultas, así como un caso en que se eligió docentes y uno en el que participaron trabajadores administrativos, personas adultas mayores, personas con un padecimiento, y turistas huéspedes de un hotel.

En relación con los aspectos bioéticos en general, más de la mitad de las investigaciones $\left(f_{i}=17\right)$ mencionaban en la metodología los aspectos bioéticos considerados para la realización de la tesis; sin embargo, en nueve no se incluyó en este apartado, y en dos no se brinda ninguna información sobre este tema.

Entre los que documentaron los aspectos bioéticos $\left(f_{i}=17\right)$ se refirieron al principio de autonomía, cuatro al de beneficencia, seis al de justicia y dos al de no maleficencia; de igual modo, en algunos trabajos se citó la confidencialidad de los datos $\left(f_{i}=13\right)$; en dos, el aseguramiento de la privacidad; en dos, se habló sobre la divulgación de los datos y en uno, acerca del asesoramiento para el proceso. Solo en cinco casos se menciona que hubo problemas para salvaguardar la bioética en el desarrollo de la tesis.

En cuanto al CI, en la mayoría de los trabajos revisados $\left(f_{i}=22\right)$, aparece incluido en los anexos, y en seis casos no se adjuntó. En 15 casos se utilizó el CI de la Vicerrectoría de Investigación de la UCR, o bien de otra fuente documentada. 
A pesar de que en un poco menos de la mitad $\left(f_{i}=11\right)$ participaron como población meta los niños, niñas o grupos vulnerables (persona adulta mayor, adolescentes, personas privadas de libertad), tan solo en cinco de ellos se presentó la adaptación del CI para esos grupos.

Al igual que en la modalidad de la práctica dirigida, en dos informes de investigación se solicitó el consentimiento de manera verbal únicamente; en otros dos informes se observa un consentimiento escueto e incluido dentro del encabezado para introducir el instrumento de recolección de datos, Finalmente, en tres de ellos no se menciona el $\mathrm{CI}$ en todo el trabajo, y en uno se menciona que el Comité hospitalario lo consideró innecesario, debido a que solo se revisaría expedientes, sin tener contacto con los (as) usuarios (as).

Según modalidad de mayor preferencia del estudiantado, el Seminario es la tercera opción más elegida como modalidad de graduación. En la tabla 4 se muestra los lugares escogidos para realizar la investigación.

\section{Tabla 4}

\section{Lugares donde se llevaron a cabo los Seminarios 2005-2012}

\begin{tabular}{lc}
\hline \multicolumn{1}{c}{ Lugar } & Frecuencia $\left(\boldsymbol{f}_{\boldsymbol{i}}\right)$ \\
Centros de I y II niveles de atención & 8 \\
Instituciones educativas & 5 \\
Hospitales de la C.C.S.S. & 3 \\
Centros de rehabilitación en adicciones & 3 \\
Programas para la atención de la PAM & 1 \\
Centros de reclusión & 1 \\
Red Social Facebook & 1 \\
Asociación Lucha contra el cáncer infantil & 1 \\
TOTAL $\left(\mathbf{F}_{\mathbf{i}}\right)$ & $\mathbf{2 3}$ \\
\hline
\end{tabular}

Fuente: elaboración propia.

Al igual que en las anteriores modalidades, los hospitales y los centros de I y II nivel de atención representan los lugares donde se realizaron las investigaciones de los seminarios : un poco menos de la mitad de los trabajos de investigación realizados $\left(f_{i}=11\right)$ se ejecutaron ahí, seguido en orden de importancia por las instituciones educativas $\left(f_{i}=5\right)$, además de que se destaca la inclusión de un grupo no incluido en las modalidades previas como es el espacio virtual de Facebook.

En cuanto a la población meta seleccionada para los seminarios, de los $\mathrm{F}_{\mathrm{i}}=23$ trabajos, la mitad $\left(f_{i}=12\right)$ agruparon como grupo etario a niños y adolescentes con y sin escolaridad; poco menos de la mitad $\left(f_{i}=7\right)$ incluyó personas adultas; tres seminarios incluyeron grupos familiares; en dos se investigó acerca de funcionarios (as) institucionales; uno abarcó docentes de primaria, dos realizaron la indagatoria con personal profesional y no profesional de enfermería; tres incluyeron personas adultas con alguna patología, personas adultas mayores, adscritas a un área de salud, y finalmente, la en una se incluyó a toda la población costarricense mediante la red social Facebook. 
Se debe destacar que de los $\mathrm{F}_{\mathrm{i}}=23$ trabajos en la modalidad de graduación de Seminario, la mitad de ellos $\left(f_{i}=12\right)$ incluyó un apartado sobre aspectos bioéticos en la metodología, nueve no lo hicieron y dos no tienen ninguna información sobre estos.

Dentro de los aspectos bioéticos considerados están el principio de autonomía $\left(f_{i}=13\right)$, el de beneficencia $\left(f_{i}=8\right)$, el de no maleficencia $\left(f_{i}=6\right)$, el de justicia $\left(f_{i}=6\right)$. Otros como la confidencialidad aparecen mencionados en ocho de ellos; en cinco se refieren a la divulgación de resultados; en cinco a la autorización para llevar a cabo el seminario por parte de las instituciones implicadas, dos mencionan el respeto a las personas con las que se trabaja, uno a la integridad y también uno al asesoramiento que se le dará a las personas en el proceso. En general, en los trabajos que cuentan con ese apartado describen bien los aspectos bioéticos.

En el caso del consentimiento informado en particular, se menciona en $f_{i}=14$ de los seminarios, pero aparece en anexos solo en $f_{i}=10$ de ellos. En tres casos, el CI es solo un encabezado en el instrumento de recolección de datos y, mientras que en cuatro trabajos se especifica que el CI fue solicitado de manera verbal. El CI de la Vicerrectoría de Investigación o de otra fuente aparece en $f_{i}=10$ de los seminarios; sin embargo, en $f_{i}=13$ casos no se menciona el origen. Solo en cuatro casos se modificó el CI para adaptarlo a poblaciones vulnerables o niños (as), aun cuando siete trabajos así lo requerían .

Finalmente, en la modalidad menos elegida está el Proyecto. En la tabla 5 se muestra los lugares donde se desarrolló las investigaciones, aunque cabe recalcar que se mantiene el patrón de ubicación en los primeros lugares los hospitales y centros de salud de la $\operatorname{CCSS}\left(f_{i}=7\right)$, seguido por los centros de salud privados $\left(f_{i}=3\right)$.

\section{Tabla 5}

\section{Lugares donde se llevaron a cabo los proyectos 2005-2012}

\begin{tabular}{lc}
\hline \multicolumn{1}{c}{ Lugar } & Frecuencia $\left(\boldsymbol{f}_{\boldsymbol{i}}\right)$ \\
Hospitales C.C.S.S. & 4 \\
Centros o programas de la C.C.S.S. & 2 \\
Centros de salud privados & 3 \\
Colegio de Enfermeras & 1 \\
Municipalidades & 1 \\
Universidad de Costa Rica & 1 \\
Museo de los Niños & 1 \\
Comisión Nacional de Emergencias & 1 \\
TOTAL $\left(\mathbf{F}_{\mathbf{i}}\right)$ & $\mathbf{1 4}$ \\
\hline
\end{tabular}

Fuente: elaboración propia.

En la mayoría de los proyectos la población meta está constituida por los profesionales de enfermería $\left(f_{i}=7\right)$; en tres fueron personas con algún padecimiento; en dos casos familiares de personas con padecimientos; un caso de padres y madres de bebés; uno con personal médico; uno con trabajadores municipales, uno con niños y niñas, uno con integrantes de una comunidad, y otro con un grupo de expertos. 


\section{.}

En cuanto a los aspectos bioéticos en la investigación, del total de proyectos, la mayoría $\left(f_{i}=10\right)$ no los incluyeron en la metodología, y solo lo hicieron cuatro de ellos. Los principios bioéticos considerados son: autonomía $\left(f_{i}=2\right.$ casos), la justicia $\left(f_{i}=2\right)$ y la beneficencia en un caso. La confidencialidad fue mencionada en 12 casos y la autorización del lugar para realizar el proyecto se menciona en $f_{i}=7$ de los trabajos. Otros aspectos mencionados en un caso cada uno fueron la revisión científica apropiada, el respeto y la divulgación de la información.

El CI únicamente aparece en los anexos de ocho proyectos; el CI de la Vicerrectoría de Investigación de la UCR solo en cuatro se utiliza, y del total solo un CI estuvo adaptado para niños y niñas. En tres casos no se menciona la presencia del CI en ninguna parte del proyecto, $\mathrm{y}$ en tres se reduce a un encabezado que introduce el instrumento de recolección de datos.

\section{DISCUSIÓN}

Antes de comenzar este apartado, es necesario aclarar que mantiene el mismo hilo conductor de la presentación de los resultados; es decir, se iniciará con las modalidades de TFG, seguida por los lugares de realización, los grupos poblacionales elegidos, las consideraciones bioéticas y finalmente el uso del CI. En lo que respecta a la modalidad de graduación, según la Universidad de Costa Rica (1980), el estudiantado tiene la posibilidad de concretarla mediante cuatro modalidades: Tesis de graduación, Seminario de graduación, Proyecto de graduación o Práctica dirigida de graduación.

Como se observó en el apartado anterior la modalidad de graduación que ocupa el primer lugar de elección durante los años analizados fue la práctica dirigida, la cual, según lo descrito por el reglamento de Trabajos Finales de Graduación (Universidad de Costa Rica, 1980), "consiste en la aplicación por parte del estudiante del conocimiento teórico de su especialidad en instituciones o empresas públicas o privadas siempre que la escuela respectiva apruebe modalidad, lugar, grupo de poblacionales" (artículo 18, p.3).Se considera que la preferencia por este tipo de modalidad se debe a su semejanza con el trabajo realizado por el alumnado en sus prácticas clínicas para dar respuestas a necesidades encontradas en poblaciones específicas, que responde a la acción de enfermería en la relación con el otro: "en un sentido genérico se refiere a aquellos actos de asistencia, de soporte o facilitadores, que son dirigidos a otro ser humano o grupo con necesidades o potenciales, con el fin de mejorar o aliviar las condiciones de la vida humana" (p.48), (Pinto, 2001) es decir, en la relación del estudiante con el Otro ${ }^{1}$, trata de dar respuesta a las demandas o necesidades encontradas que guían no solo en el crecimiento del arte de cuidar, sino también en el crecimiento de quien cuida y es cuidado (Mayeroff ${ }^{2}, 1990$, Noddings ${ }^{3}, 1988$ ).

En relación con lo anterior, el Plan de estudio actual establece el cuidado como el objeto de estudio de la enfermería y agrega en cuanto al abordaje de este, que

\footnotetext{
1 Ese Otro con mayúscula porque se comprende como el ser humano, individual, en grupo social o cultural.

${ }^{2}$ Fue uno de los primeros en explorar el concepto filosófico del cuidado: en su libro, On Caring, menciona que el cuidado es esencialmente humano, que ayudando al otro y a su vez, se ayuda a uno mismo a crecer.

3 Noddings, es otra destacada teórica del cuidado, con un enfoque filosófico de género dirigido a la educación de la enfermería.
} 
el abordaje del objeto de conocimiento (objeto material y objeto formal) es posible desde el punto de vista de la unidad entre la teoría y experiencia. Es decir, es posible el abordaje del objeto de conocimiento, de los campos de acción, a partir de la unidad de discusión teórica y de la experiencia concreta, es que con frecuencia se denomina "práctica". Los discursos teóricos permiten comprender o explicar la experiencia, la realidad concreta con la cual se enfrenta un saber (Escuela de Enfermería, 1997, p. 13).

Como se evidencia en la cita anterior, la denominada práctica ha sido no solo la forma de hacer de la enfermería, sino la de aprender; por tanto, la modalidad de graduación de práctica de enfermería es adecuada para el estudiantado en cuanto a que le ofrece la posibilidad de aplicar el conocimiento teórico adquirido en su especialidad a grupos determinados.

Si bien es cierto, por un lado es positivo que el estudiantado elija como modalidad de graduación el dar respuesta a la necesidad de cuidado de un grupo poblacional determinado, por otro, refleja la separación que por mucho tiempo ha tenido la investigación de la práctica y la enseñanza de la enfermería, como lo expresan Orellana y Sanhueza (2011), cuando tratan de explicitar la importancia de la investigación en enfermería:

La investigación es una función ineludible del profesional de enfermería, poco valorada por las instituciones de salud, y poco exigida en el actuar de enfermería y más aún, colocada en último plano por el mismo profesional de enfermería. Las principales razones empíricas pueden ser: una baja conciencia de la importancia de la investigación, la creencia de que es un área compleja, la sobrecarga de trabajo, así como también la falta de curiosidad, perpetuando el siempre se ha hecho así (p.11).

Lo expresado en cita fue encontrado en el año 1997 cuando se realizó el estudio para transformar el bachillerato en enfermería en Licenciatura, respecto de lo que se detectó que

Aun cuando las enfermeras admiten poseer conocimientos básicos de investigación, la realidad en cuanto a su aplicación en la práctica, indica que esta no se da, o se da de forma esporádica y ocasional. Podría significar esto que los principios de la investigación se conocen en términos teóricos y memorísticos, sin que se haya incorporado como una forma de enfrentar los problemas que la práctica impone; ni tampoco se le considera una actitud que permite el desarrollo de la disciplina, de modo que nutra la teoría y se vea, a su vez, definida y orientada desde esta (Escuela de Enfermería, (1997,p.109).

Dado lo anterior, se decidió incorporar la investigación como un eje transversal del nuevo plan de estudios, lo cual explicaría por qué las modalidades de tesis y seminarios ocupan el segundo lugar y un creciente número en los años estudiados, debido a que ambas incluyen un proceso investigativo.

El Reglamento de trabajos finales de graduación, en su artículo siete, define la Tesis de Graduación como "un proceso de investigación que culmina con un trabajo escrito que aporta algo original sobre el asunto investigado" (p.1). Acerca del Seminario de Graduación y en el artículo 11 se define como 


\section{.}

una actividad académica [...] que se ofrece a lo largo de uno, dos o tres ciclos consecutivos, como máximo, a un grupo de estudiantes [...] quienes, mediante su participación reiterada alrededor de algún problema científico o profesional, se familiarizan con las teorías y métodos de investigación propios de la disciplina y su aplicación a casos específicos bajo la guía del director del trabajo (Universidad de Costa Rica,1980, p.2).

En ambas modalidades se refieren a un proceso científico riguroso, cuyo fin es aportar resultados o hallazgos para las diferentes áreas del saber, así lo expone Caro (2009) al afirmar que "claramente la investigación científica constituye un instrumento valioso para exploración, indagación, análisis y solución de problemas de naturaleza práctica, enmarcados en diferentes áreas del saber constituido de enfermería" (p. 12). Sin embargo, para poder comprender los aspectos de bioética que se analizarán posteriormente es imprescindible que estos procesos en los que se inscribe el estudiantado sean guiados por los profesores, encargados no solo de conocer los tipos y enfoques del proceso investigativo, sino los aspectos éticos fundamentales como los bioéticos, inherentes a toda investigación que involucre seres humanos, una labor institucional que no debe obviarse, máxime que "en razón de que la mayoría de los procesos investigativos son desarrollados desde la academia, es a esta a quien le corresponde legitimar lo que hace, mostrar la pertinencia y el merito social de sus productos investigativos" (Caro, 2009, p.12).

La academia debe marcar la pauta y modelar conductas, comportamientos esperados en diversas áreas del saber propio de la disciplina, como en el caso de la investigación. Si a lo anterior aunamos que, en muchos casos, los grupos poblacionales meta de las investigaciones realizadas son denominados vulnerables ${ }^{1}$, es imprescindible que en los informes finales de tesis y seminarios de graduación se muestre de forma explícita y exhaustiva todo aspecto necesario para salvaguardar los derechos de las personas participantes y que además, como aporta Garrafa (2005), la ética en la academia se convierta en una herramienta para generar propuestas para el bienestar de las personas y comunidades.

Pese a lo mencionado, en el apartado de resultados se puede constatar que la gran mayoría de los informes finales de investigación no muestra ${ }^{2}$ exhaustividad en las consideraciones bioéticas descritas por diversas organizaciones (UNESCO, 2005; Informe Belmont, 1976; Consejo de Organizaciones de las Ciencias Médicas, 2002; Declaración de Helsinki, 2013; Universidad de Costa Rica, 2011; Ley Reguladora de Investigación Biomédica, 2014), las cuales no deben ser obviadas durante los procesos investigativos.

El explicitar dicha información no es vinculante con el desarrollo de los trabajos; sin embargo, se debe comprender que los estudiantes que elaboran un Trabajo Final de Graduación, aun en este nivel final de la formación profesional, aun se encuentran en un proceso de aprendizaje en el que se les debe recalcar la trascendencia de incorporar las regulaciones que protegen a los seres humanos que participan en investigaciones, máxime que, como lo menciona León (2008)

\footnotetext{
${ }^{1}$ Según Pérez (2005) "se entiende por grupos vulnerables a todos aquellos que, ya sea por su edad, raza, sexo, condición económica, características físicas, circunstancia cultural o política, se encuentran en mayor riesgo de que sus derechos sean violentados (p.846)

2 Esto no debe interpretarse que no se realizó en la ejecución del trabajo investigativo.
} 
la formación en bioética es parte de la necesaria competencia del profesional de la salud, cuyo objetivo es proporcionar elementos de juicio a los futuros profesionales para comprender los debates en bioética que se producen en su sociedad (p.12).

Comentado lo anterior, se aborda ahora el tema de las poblaciones metas de los estudios realizados que, en su gran mayoría, son grupos en situación de vulnerabilidad, ya sea personas privadas de libertad, personas menores de edad y adolescentes, personas con capacidad disminuida transitoria o permanente.

Para definir brevemente el concepto de vulnerabilidad, se empleará lo mencionado por Pineda y Magaña (2013), quienes comentan que "los llamados grupos vulnerables no son vulnerables per se, sino que son las condiciones sociales, económicas y políticas las que definen su condición de vulnerabilidad; por lo que, de manera precisa, deben ser denominados grupos en situación de vulnerabilidad" (p.14). Por las características de dichos grupos, se aplica, desde el punto de vista de la bioética, el respeto a la vulnerabilidad, la cual

debe verse como un principio biopolítico medular del estado de bienestar moderno [...] sin embargo, el alcance es más amplio que el fomento de la vulnerabilidad como la expresión fundamental y universal de la condición humana; apela a la protección de la vida- de las bioien general [...] por tanto, el respeto por la vulnerabilidad debe ser reconocido no solo como el principio más esencial de la bioética sino como la base de toda la ética (Helge, 2011 p. 91).

Como se observa en la cita $\mathrm{r}$, la consideración de la vulnerabilidad es una observación apremiante de que considera en las intervenciones e investigaciones; su relevancia es tal, que en el artículo 8 de la Declaración de los Derechos humanos se menciona que "los individuos y grupos especialmente vulnerables deberían ser protegidos y se debería respetar la integridad personal de dichos individuos", afirmación que fundamenta la idea que de que todo trabajo investigativo de los estudiantes debe mostrar rigurosidad en la forma en que se protegió a los grupos vulnerables o en condición de vulnerabilidad con los que se trabajó el TFG.

Un elemento que muestra claramente la forma en que se manifiestan los principios bioéticos y el respeto y protección de los grupos en condición de vulnerabilidad es el Consentimiento Informado que, para el presente artículo, es un proceso que va más allá de la firma de un documento estructurado, sino que conlleva un proceso en que se brinda suficiente información para tomar decisiones con conocimiento, hecho por el que es necesario emplear un lenguaje accesible, sencillo, directo y comprensible, en el que prevalece una relación dialógica, empática con un genuino respeto de quien investiga, por ende, su validez depende que incluya los siguientes elementos básicos (Castellanos, López, Caballé y García, 2009)

El proceso de consentimiento informado debe ser realizado por la persona/familiar/ tutor de forma libre, voluntaria y sin presiones de ningún tipo.

La persona/ familiar/ tutor debe (n) tener información suficiente de parte del o la investigadora, para tomar una decisión sustentada adecuadamente.

El proceso de consentimiento informado debe gestarse en una relación simétrica externada en la forma dialógica y escrita. 
El proceso de consentimiento informado debe darse con antelación suficiente al desarrollo del trabajo.

La persona, familiar o tutor que autoriza el consentimiento informado debe tener competencia o capacidad suficiente para comprender lo que va a asentir.

Si la persona es definida como un sujeto en condición de vulnerabilidad, quien lo represente no debe serlo.

El documento de consentimiento informado debe expresar incuestionable y formalmente la legalidad existente sobre esta temática.

De acuerdo con lo anterior, es fundamental, apremiante, ético y legal, que se explicite con claridad el proceso que se realizó con las poblaciones objeto de estudio, para salvaguardar la validez del trabajo, todo sustentado en la siguiente afirmación "las circunstancias de la humanidad, cambiantes y tan diversas orientan la exigencia de la construcción de un saber y una práctica bioética" (p.100) (Garrafa, Kottow y Saada, 2005).

\section{CONCLUSIONES}

- La modalidad de trabajo final de graduación por la que optó mayormente el estudiantado, entre los años 2005 al 2012, fue la Práctica Dirigida, que responde muy bien al enfoque pragmático de la enfermería de esos años.

- La elección de las modalidades de graduación de Tesis y Seminarios, parece responder a la intensa entrada de la investigación como eje transversal en el programa de estudios.

- Esta inserción más lenta explicaría las debilidades de explicitar los aspectos bioéticos incluidos en la investigación.

- Es interesante que a pesar del porcentaje de trabajos que no incluyen aspectos o fundamentación bioéticos, los que sí lo hacen, en todos los casos, consideran aspectos relacionados únicamente con la corriente del Principialismo.

- No se explicitan los aspectos tomados en cuenta en la generación del documento de consentimiento informado.

\section{AGRADECIMIENTO}

Un agradecimiento especial a la investigadora Karol Blanco Rojas, que colaboró en el agrupamiento de la información. 


\section{Revista Electrónica Enfermería Actual de costa Rica}

\section{REFERENCIAS BIBLIOGRAFICAS}

Caro, C (2009). Papel de la investigación en enfermería. Revista avances en enfermería. 2009; XXVI (2);11-12 [Revista en Línea] Recuperado de: http://www.enfermeria.unal.edu.co/revista/articulos/xxvii2 1.pdf

Carper, B. (1978). Fundamental patterns of knowing in nursing, Advances in Nursing Science, 1 (1) 13-24.

Castellanos, M., López, J, Caballé, M., García, H. (2009). El consentimiento informado; una acción imprescindible en la investigación médica. Rev. Cubana Estomatol. Mar; 46(1) Recuperado de:

http://scielo.sld.cu/scielo.php?script=sci arttext\&pid=S0034-75072009000100007\&lng=es.

Consejo de Organizaciones Internacionales de las Ciencias Médicas (2002). Pautas éticas internacionales para la investigación biomédica en seres humanos. Ginebra: CIOMS. Recuperado de http://cioms.ch/publications/guidelines/pautas eticas internacionales.htm

Declaración de Helsinki (2013). Principios éticos para las investigaciones médicas en seres humanos. Brasil: Asamblea general. Fortaleza, octubre, 2013. Recuperado de http://www.wma.net/es/30publications/10policies/b3/

Daza, R., Medina, L. (2006). Significado del cuidado de enfermería desde la perspectiva de los profesionales de una institución hospitalaria de tercer nivel en Santa Fe de Bogotá, Colombia. Rev. Cultura de los Cuidados. Año X, 19, 55-62.

Durán, M. (2002) Marco epistemológico de la enfermería. Rev. Aquichan. Universidad de La Sabana. 2(1) 7-18.

Escuela de Enfermería (1997). Tomo I: Reestructuración del Bachillerato que se transforma en la Licenciatura en Enfermería. Resolución $N^{\circ}$ 6479-98. Costa Rica: Universidad de Costa Rica.

Garrafa, V. (2005). Da bioética de principios a una bioética interventiva. Bioética, 13(1)125-134.

Garrafa, V., Kottow, M., Saada, A. (2005) Estatuto Epistemológico de la Bioética. UNAM. Red Latinoamericana y del Caribe de Bioética de UNESCO, 1, 314 p.

Garrafa, V. (1999).Bioética, Saúde e Ciudadania. O Mundo Da Saúde, 23(5) 263- 269.

Garrafa, V., Do Prado, M. (2001). Cambios en la Declaración de Helsinki: Fundamentalismo económico, imperialismo ético y control social. Jurisprudencia Argentina, (IV) 11: 09-15.

Gracia, D. (1989). Fundamentos de Bioética. Madrid: Eudema.

Helge, J. (2011). Vulnerabilidad: ¿un principio fútil o útil en la ética de la asistencia sanitaria? Rev. Redbioética/UNESCO, año2, 1 (3), p. 89-101, enero- junio. Recuperado de: http://unesco.org.uy/ci/fileadmin/shs/redbioetica/revista 3/Solbakk.pdf

Hottois, G. (2007). ¿Qué es la Bioética? Bogota: Universidad El Bosque. 
Informe Belmont (1976). Principios éticos y directrices para la protección de sujetos humanos de investigación: Reporte de la Comisión Nacional para la Protección de Sujetos Humanos de Investigación Biomédica y de Comportamiento.

Washington: Secretaría de Salud, Educación y Bienestar social. Recuperado de https://etsu.edu/irb/Belmont\%20Report\%20in\%20Spanish.pdf

La Gaceta. (2014). Ley Reguladora de la Investigación Biomédica. Costa Rica: Asamblea Legislativa de la República de Costa Rica. Recuperado de www.gaceta.go.cr/pub/2014/04/25/COMP 2504 2014.pdf

León, F. (2008). Enseñar bioética: cómo trasmitir conocimientos, actitudes y valores. Acta bioethica, 14(1), 11-18. Recuperado de http://dx.doi.org/10.4067/S1726-569X2008000100002

Mayeroff, M. (1990). On Caring. New York: Harper Collins.

Noodings, N. (1988) An ethic of caring and its implications for instructional arrangements. American Journal of Education, 96 (2) 215-230.

Orellana, A., Sanhueza, O. (2011). Competencia en Investigación en enfermería. Ciencia y enfermería, 17(2), 9-17. Recuperado de: http://dx.doi.org/10.4067/S0717-95532011000200002

Pérez, M. (2005). Aproximación a un estudio sobre vulnerabilidad y violencia familiar. Boletín Mexicano de Derecho Comparado, año XXXVIII, ${ }^{\circ} 113$, mayo- agosto, pp. 845-867. Recuperado de: http://biblio.juridicas.unam.mx/revista/pdf/DerechoComparado/113/art/art9.pdf

Pineda, G., Magaña, M. (2013). Aproximación bioética al estudio de la vulnerabilidad social. Regiones, Suplemento de Antropología, 49, 13-17. Recuperado de: http://suplementoregiones.com/? $\mathrm{p}=543$

Pinto, N. (2002). El Cuidado como objeto del conocimiento de enfermería. Rev. Avances de Enfermería, vol. XIX, 2 , $43-51$. Facultad de Enfermería: Universidad Nacional de Colombia.

Sanhueza, O. (2009). Contribución de la investigación cualitativa a enfermería. Ciencia y enfermería, 15(3), 15-20. Recuperado de: http://dx.doi.org/10.4067/S0717-95532009000300003

Torre, E., Navarro, A. (1982). Metodología de la investigación: bibliográfica, archivística y documental. México D. F.: McGraw Hill.

UNESCO (2005). Declaración Universal sobre Bioética y Derechos Humanos. Washington: Organización de las Naciones Unidas para la Educación, la Ciencia y la Cultura. Recuperado de: http://www.unesdoc.unesco.org/images/0014/001461/146180s.pdf

Universidad de Costa Rica (2011). Resolución de la Vicerrectoría de Investigación sobre investigaciones con seres humanos: Resolución VI-1922-2011. Costa Rica: Universidad de Costa Rica. Recuperado de: www.vinv.ucr.ac.cr/index.php?option=com_docman\&task

Universidad de Costa Rica (1980). Reglamento de Trabajos Finales de Graduación. Recuperado de: http://www.cu.ucr.ac.cr/normativ/trabajos finales graduacion.pdf 


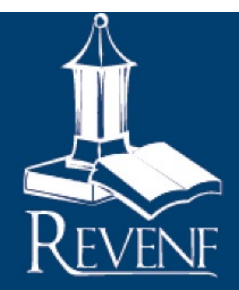

Revista Electrónica Enfermeria Actual de costa Rica www.revenf.ucr.ac.cr

Vidal, S. (2010). Bioética y desarrollo humano: una visión desde América Latina. Rev. Redbioética/UNESCO, 1(1), 81-123. 